\title{
Quantization of strings without anomaly in spacetime of arbitrary dimensionality
}

\begin{abstract}
Zahid Zakir *
Abstract

The new method of $C$-symmetric quantization, strongly following to the restrictions of discrete symmetries of systems, is applied to quantization of closed boson strings. It is shown that the chiral symmetry restrictions exclude a zero-point energy of the string modes. It then leads to the theory of the relativistic strings without conformal anomaly, which can be quantized consistently in a spacetime of arbitrary dimensionality.
\end{abstract}

PACS: 11.25.-w, 12.10.-g,

Key words: strings, quantization, conformal anomaly, discrete symmetries

\section{Content}

Introduction 9

1. Closed boson string without zero-point energy of modes.................................... 10

2. Boson string without anomaly in spacetime of arbitrary dimensionality ................. 13

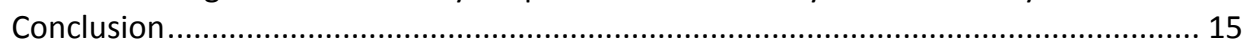

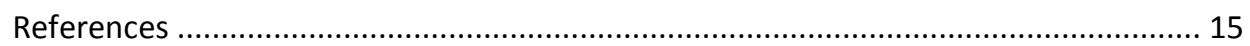

\section{Introduction}

The standard quantum theory of local fields, where fundamental particles appear as quanta of these fields, contains divergences which did not be able to excluded long time, especially at including of gravity. As a result, more radical approaches based on introduction of nonlocal fundamental objects - strings and branes - have been proposed and widely developed.

Distinctive feature of the string theory is a highly exclusive role which plays the zero-point energy of oscillatory modes of these nonlocal structures. Just the zeropoint energy leads to an anomaly and then to a central extension of the Virasoro algebra in the string theory and a condition of disappearing of this anomaly allows then to fix a dimensionality of full spacetime that further admits only a restricted class of states and gauge symmetries. The existence of such strong restrictions has been considered as a great advantage of these approaches and initiated large enthusiasm.

In the previous papers [1] the new method of quantization with strong obeying to the restrictions of charge-conjugation $(C)$ symmetry has been developed and it has been shown that in the system with these symmetries a zero-point energy does not arise. In case of the photon field, for example, it is related by the conserving helicity, which in fact is a chiral charge of photon, and there vectors of electrical and magnetic

*Centre for Theoretical Physics and Astrophyics, Tashkent, Uzbekistan; zahidzakir@theor-phys.org 
field of photon do not vibrate, but rotate on a plane that analogous to the soft rotators also not having a zero-point energy.

In the present paper this new quantization method, more strongly taking into account requirements of discrete symmetries, is applied to quantization of closed boson strings. In section 1 it is shown that the chiral symmetry excludes a zero-point energy of string modes. In section 2 it is shown that this leads to the theory of the relativistic strings without conformal anomaly, which can be consistently quantized in the spacetimes of arbitrary dimensionality.

\section{Closed boson string without zero-point energy of modes}

Let's consider standard action for boson strings:

$$
S=-\frac{T}{2} \int d \tau d \sigma h^{\alpha \beta}(\tau, \sigma) \partial_{\alpha} X^{\mu} \partial_{\beta} X_{\mu} .
$$

Three local symmetries, two reparametrizations and the Weyl dilatation allow choosing three independent components of the 2-metric of the string as:

$$
h_{\alpha \beta}(\tau, \sigma)=\eta_{\alpha \beta}=\left(\begin{array}{cc}
1 & 0 \\
0 & -1
\end{array}\right),
$$

Then the action (1) can be written in the form:

$$
S=-\frac{T}{2} \int d^{2} \sigma \eta^{\alpha \beta} \partial_{\alpha} X^{\mu} \partial_{\beta} X_{\mu} .
$$

Equations of motion for the closed string, following from this action:

$$
\partial_{\alpha} \partial^{\alpha} X^{\mu}=\left(\partial_{\tau}^{2}-\partial_{\sigma}^{2}\right) X^{\mu}=0
$$

have a general solution in the form of superposition of two independent modes:

$$
X^{\mu}(\tau, \sigma)=X_{R}^{\mu}(\tau-\sigma)+X_{L}^{\mu}(\tau+\sigma) .
$$

Coordinates of these two modes can be joined to a complex coordinate:

$$
\begin{aligned}
& Y^{\mu}(\tau, \sigma)=\frac{1}{\sqrt{2}}\left[X_{R}^{\mu}(\tau-\sigma)+i X_{L}^{\mu}(\tau+\sigma)\right], \\
& Y^{\mu^{*}}(\tau, \sigma)=\frac{1}{\sqrt{2}}\left[X_{R}^{\mu}(\tau-\sigma)-i X_{L}^{\mu}(\tau+\sigma)\right] .
\end{aligned}
$$
a form:

In terms of this coordinate the action and the energy-momentum tensor take

$$
\begin{gathered}
S=-\frac{T}{2} \int d^{2} \sigma \eta^{\alpha \beta}\left[\partial_{\alpha} X_{R}^{\mu} \partial_{\beta} X_{\mu R}+\partial_{\alpha} X_{L}^{\mu} \partial_{\beta} X_{\mu L}\right]= \\
=-T \int d^{2} \sigma \eta^{\alpha \beta} \partial_{\alpha} Y^{*} \cdot \partial_{\beta} Y, \\
T_{\alpha \beta}=-\frac{2}{T \sqrt{-\eta}} \frac{\delta S}{\delta \eta^{\alpha \beta}}= \\
=\frac{1}{2}\left[\partial_{\alpha} Y_{\mu}^{*} \partial_{\beta} Y^{\mu}+\partial_{\beta} Y_{\mu}^{*} \partial_{\alpha} Y^{\mu}-\frac{1}{2} \eta_{\alpha \beta} \eta^{\alpha^{\prime} \beta^{\prime}}\left(\partial_{\alpha^{\prime}} Y_{\mu}^{*} \partial_{\beta} Y^{\mu}+\partial_{\beta} Y_{\mu}^{*} \partial_{\alpha} Y^{\mu}\right)\right]=0 .
\end{gathered}
$$

The traceless of the energy-momentum tensor leads to the constraint equations: 


$$
\begin{aligned}
& T_{00}=T_{11}=\frac{1}{2}\left(\partial_{\tau} Y^{*} \partial_{\tau} Y+\partial_{\sigma} Y^{*} \partial_{\sigma} Y\right)=0, \\
& T_{10}=T_{01}=\frac{1}{2}\left(\partial_{\tau} Y^{*} \cdot \partial_{\sigma} Y+\partial_{\sigma} Y^{*} \cdot \partial_{\tau} Y\right)=0,
\end{aligned}
$$

which give the relations:

$$
\begin{aligned}
& \partial_{\tau} Y^{*} \partial_{\tau} Y=\partial_{\sigma} Y^{*} \partial_{\sigma} Y=0, \\
& \partial_{\tau} Y^{*} \cdot \partial_{\sigma} Y=\partial_{\sigma} Y^{*} \cdot \partial_{\tau} Y=0 .
\end{aligned}
$$

The equations of motion (4) and the boundary conditions for the closed strings:

$$
\begin{aligned}
& Y^{\mu}(\tau, \sigma)=Y^{\mu}(\tau, \sigma+2 \pi), \\
& Y^{\mu^{*}}(\tau, \sigma)=Y^{\mu^{*}}(\tau, \sigma+2 \pi),
\end{aligned}
$$

lead to following decomposition of the complex coordinate:

$$
\begin{aligned}
& Y^{\mu}(\tau, \sigma)=\frac{y^{\mu}}{2}+\frac{l^{2}}{2}\left(\pi^{\mu} \tau-\pi^{\mu^{*}} \sigma\right)+\frac{i l}{2} \sum_{n \neq 0} \frac{1}{|n|}\left(\alpha_{n}^{\mu} e^{-2 i(|n| \tau-n \sigma)}+b_{n}^{\mu^{*}} e^{2 i(|n| \tau-n \sigma)}\right), \\
& Y^{\mu^{*}}(\tau, \sigma)=\frac{y^{\mu^{*}}}{2}+\frac{l^{2}}{2}\left(\pi^{\mu^{*}} \tau-\pi^{\mu} \sigma\right)-\frac{i l}{2} \sum_{n \neq 0} \frac{1}{|n|}\left(\alpha_{n}^{\mu^{*}} e^{2 i(|n| \tau-n \sigma)}+b_{n}^{\mu} e^{-2 i(|n| \tau-n \sigma)}\right),
\end{aligned}
$$

where $l^{2}=1 / \pi T$, and

$$
y^{\mu}=\frac{1+i}{\sqrt{2}} x^{\mu}, \quad \pi^{\mu}=\frac{1-i}{\sqrt{2}} p^{\mu} .
$$

This gives also the expressions:

$$
\begin{aligned}
& \partial_{\tau} Y^{\mu}(\tau, \sigma)=\frac{1}{2} l^{2} \pi^{\mu}+l \sum_{n \neq 0}\left(\alpha_{n}^{\mu} e^{-2 i(|n| \tau-n \sigma)}-b_{n}^{\mu^{*}} e^{2 i(|n| \tau-n \sigma)}\right), \\
& \partial_{\tau} Y^{\mu^{*}}(\tau, \sigma)=\frac{1}{2} l^{2} \pi^{\mu^{*}}+l \sum_{n \neq 0}\left(\alpha_{n}^{\mu^{*}} e^{2 i(|n| \tau-n \sigma)}-b_{n}^{\mu} e^{-2 i(|n| \tau-n \sigma)}\right),
\end{aligned}
$$

A difference from a standard form is that the proper times $\tau$ on worldsheets are multiplied only to the positive "frequencies" $|n|$. Therefore the sums of two independent contributions of opposite sign of frequency are written separately, where creation operators of positive-frequency quanta are denoted as $\alpha_{n}^{\mu^{*}}$, while the auxiliary operators for negative-frequency quanta as $b_{n}^{\mu^{*}}$.

The equal time commutators look like:

$$
\begin{aligned}
& i\left[\partial_{\tau} Y^{\nu}(\tau, \sigma), Y^{\mu^{*}}\left(\tau, \sigma^{\prime}\right)\right]=-\frac{1}{T} \eta^{\mu \nu} \delta\left(\sigma-\sigma^{\prime}\right), \\
& i\left[\partial_{\tau} Y^{\nu^{*}}(\tau, \sigma), Y^{\mu}\left(\tau, \sigma^{\prime}\right)\right]=-\frac{1}{T} \eta^{\mu \nu} \delta\left(\sigma-\sigma^{\prime}\right) .
\end{aligned}
$$

The substitution of (12) and (13) give us following nonzero commutators for the ladder operators of modes: 


$$
\begin{aligned}
& {\left[\alpha_{m}^{\mu}, \alpha_{n}^{v^{*}}\right]=\left[b_{m}^{\mu}, b_{n}^{\nu^{*}}\right]=-|m| \eta^{\mu v} \delta_{m n},} \\
& i\left[\pi^{\mu}, y^{v}\right]=i\left[\pi^{\mu^{*}}, y^{v^{*}}\right]=-\eta^{\mu \nu},
\end{aligned}
$$

where $\alpha_{0}^{\mu}=b_{0}^{\mu}=l \pi^{\mu} / 2$.

The conserved currents for translations of coordinates $Y^{\mu}, Y^{\mu^{*}}$ are

$$
\begin{gathered}
P_{\alpha}^{\mu}=T \partial_{\alpha} Y^{\mu^{*}}, \quad P_{\alpha}^{\mu^{*}}=T \partial_{\alpha} Y^{\mu}, \\
P_{\tau}^{\mu}=\frac{1}{2 \pi} \pi^{\mu}+l \sum_{n \neq 0}\left(\alpha_{n}^{\mu} e^{-2 i(|n| \tau-n \sigma)}-b_{n}^{\mu^{*}} e^{2 i(|n| \tau-n \sigma)}\right), \\
P_{\tau}^{\mu^{*}}=\frac{1}{2 \pi} \pi^{\mu^{*}}+l \sum_{n \neq 0}\left(\alpha_{n}^{\mu^{*}} e^{2 i(|n| \tau-n \sigma)}-b_{n}^{\mu} e^{-2 i(|n| \tau-n \sigma)}\right), \\
P_{\sigma}^{\mu}=-\frac{1}{2 \pi} \pi^{\mu^{*}}-l \sum_{n \neq 0} \frac{n}{|n|}\left(\alpha_{n}^{\mu} e^{-2 i(|n| \tau-n \sigma)}-b_{n}^{\mu^{*}} e^{2 i(|n| \tau-n \sigma)}\right), \\
P_{\sigma}^{\mu^{*}}=-\frac{1}{2 \pi} \pi^{\mu}-l \sum_{n \neq 0} \frac{n}{|n|}\left(\alpha_{n}^{\mu^{*}} e^{2 i(|n| \tau-n \sigma)}-b_{n}^{\mu} e^{-2 i(|n| \tau-n \sigma)}\right), \\
\partial^{\alpha} P_{\alpha}^{\mu}=0, \quad \partial^{\alpha} P_{\alpha}^{\mu^{*}}=0 .
\end{gathered}
$$

The total momentum of the closed string can be found be integrating currents on $\sigma$ at $\tau=0$ :

$$
P^{\mu}=T \int_{0}^{2 \pi} d \sigma \partial_{\tau} Y^{\mu^{*}}=\pi^{\mu} .
$$

The Hamiltonian then takes the form:

$$
\begin{gathered}
H=\int_{0}^{2 \pi} d \sigma\left[\left(P_{\tau}^{\mu} \partial_{\tau} Y_{\mu}+\partial_{\tau} Y_{\mu}^{*} P_{\tau}^{\mu^{*}}\right)-L\right]=T \int_{0}^{2 \pi} d \sigma\left(\partial_{\tau} Y_{\mu}^{*} \partial_{\tau} Y^{\mu}+\partial_{\sigma} Y_{\mu}^{*} \partial_{\sigma} Y^{\mu}\right), \\
H=T \int_{0}^{2 \pi} d \sigma\left(\partial_{\tau} Y^{\mu^{*}} \partial_{\tau} Y_{\mu}+\partial_{\sigma} Y^{\mu^{*}} \partial_{\sigma} Y_{\mu}\right)=2 \sum_{n=-\infty}^{\infty}\left(\alpha_{n}^{\mu^{*}} \alpha_{n \mu}+b_{n}^{\mu} b_{n \mu}^{*}\right) .
\end{gathered}
$$

The left-hand and right-hand modes of the string are independent and a conserved helicity operator of the string, playing the role of a chiral charge, is given by the expressions:

$$
\begin{aligned}
\Lambda & =T \int_{0}^{2 \pi} d \sigma\left[\left(\partial_{\tau} Y_{\mu}^{*}\right) Y^{\mu}-Y^{\mu^{*}} \partial_{\tau} Y_{\mu}\right]=\int_{0}^{2 \pi} d \sigma\left(P_{\tau}^{\mu} Y_{\mu}-Y_{\mu}^{*} P_{\tau}^{\mu^{*}}\right)= \\
& =i\left(\pi^{\mu} y^{\nu}-y^{\nu^{*}} \pi^{\mu^{*}}\right)-i \sum_{n \neq 0} \frac{1}{|n|}\left(\alpha_{n}^{\mu^{*}} \alpha_{n \mu}-b_{n}^{\mu} b_{n \mu}^{*}\right) .
\end{aligned}
$$

There is a symmetry transferring the left-modes to right-modes and backward by chiral charge conjugation (C-symmetry). Let's introduce also the operators $C$ conjugate to former operators of the string modes:

$$
\begin{aligned}
& \beta_{n}^{\mu}=C \alpha_{n}^{\mu} C^{-1}, \quad \beta_{n}^{\mu^{*}}=C \alpha_{n}^{\mu^{*}} C^{-1}, \\
& a_{n}^{\mu}=C b_{n}^{\mu} C^{-1}, \quad a_{n}^{\mu^{*}}=C b_{n}^{\mu^{*}} C^{-1} .
\end{aligned}
$$


Further, by means of these operators the $C$-symmetry requirements can be written in the form:

$$
\begin{aligned}
& H=\sum_{n=-\infty}^{\infty}\left(\alpha_{n}^{\mu^{*}} \alpha_{n \mu}+b_{n}^{\mu} b_{n \mu}^{*}\right)=\sum_{n=-\infty}^{\infty}\left(\beta_{n}^{\mu^{*}} \beta_{n \mu}+a_{n P}^{\mu} a_{n \mu P}^{*}\right)=H^{c}, \\
& \Lambda=-i \sum_{n=-\infty}^{\infty} \frac{1}{|n|}\left(\alpha_{n}^{\mu^{*}} \alpha_{n \mu}-b_{n}^{\mu} b_{n \mu}^{*}\right)=i \sum_{n=-\infty}^{\infty} \frac{1}{|n|}\left(\beta_{n}^{\mu^{*}} \beta_{n \mu}-a_{n P}^{\mu} a_{n \mu P}^{*}\right)=-\Lambda^{c} .
\end{aligned}
$$

Because of independence of modes the $C$-symmetry requirements take place for each mode separately and consequently (at $\left.y^{v}=0\right)$ :

$$
\begin{aligned}
& H_{n}=\left(\alpha_{n}^{\mu^{*}} \alpha_{n \mu}+b_{n}^{\mu} b_{n \mu}^{*}\right)=\left(\beta_{n}^{\mu^{*}} \beta_{n \mu}+a_{n}^{\mu} a_{n \mu}^{*}\right)=H_{n}^{c}, \\
& \Lambda_{n}=\frac{1}{|n|}\left(\alpha_{n}^{\mu^{*}} \alpha_{n \mu}-b_{n}^{\mu} b_{n \mu}^{*}\right)=-\frac{1}{|n|}\left(\beta_{n}^{\mu^{*}} \beta_{n \mu}-a_{n}^{\mu} a_{n \mu}^{*}\right)=-\Lambda_{n}^{c},
\end{aligned}
$$

or:

$$
\begin{aligned}
& \alpha_{n}^{\mu^{*}} \alpha_{n \mu}+b_{n}^{\mu} b_{n \mu}^{*}=\beta_{n}^{\mu^{*}} \beta_{n \mu}+a_{n}^{\mu} a_{n \mu}^{*}, \\
& \alpha_{n}^{\mu^{*}} \alpha_{n \mu}-b_{n}^{\mu} b_{n \mu}^{*}=-\beta_{n}^{\mu^{*}} \beta_{n \mu}+a_{n}^{\mu} a_{n \mu}^{*} .
\end{aligned}
$$

For each string mode, as a result, we obtain the relations:

$$
\begin{aligned}
& \alpha_{n}^{\mu^{*}} \alpha_{n \mu}=a_{n}^{\mu} a_{n \mu}^{*}, \\
& \beta_{n}^{\mu^{*}} \beta_{n \mu}=b_{n}^{\mu} b_{n \mu}^{*} .
\end{aligned}
$$
form:

Thus, due to (28) the Hamiltonian and the helicity (25) of the string take a

$$
\begin{aligned}
& H=\sum_{n \neq 0}\left(\alpha_{n}^{\mu^{*}} \alpha_{n \mu}+\beta_{n \mu}^{*} \beta_{n}^{\mu}\right)=H^{c}, \\
& \Lambda=\sum_{n \neq 0} \frac{1}{|n|}\left(\alpha_{n}^{\mu^{*}} \alpha_{n \mu}-\beta_{n \mu}^{*} \beta_{n}^{\mu}\right)=-\Lambda^{c},
\end{aligned}
$$

where the operators of string modes enter in a normal-ordered form and there is no zero-point energy.

The absence of a zero-point energy leads to a quantum theory of strings without a conformal anomaly, since only the presence of the zero-point energy of the string modes was a physical reason for this anomaly. Then the lack of the anomaly does not allow to fix dimensionality of spacetime that earlier has been considered as one of main achievements of the string theories. In the following section these facts will be considered in more detail form.

\section{Boson string without anomaly in spacetime of arbitrary dimensionality}

Let's consider the decomposition on modes of the constraints $T_{\alpha \beta}=0$. For the closed string the constraints are reduced to $\partial_{\tau} Y^{*} \partial_{\tau} Y=\partial_{\sigma} Y^{*} \partial_{\sigma} Y=0$ and (at $\tau=0)$ :

$$
L_{m}=\frac{T}{2} \int_{0}^{2 \pi} d \sigma e^{-i m \sigma} T_{00}(0, \sigma)=\frac{T}{4} \int_{0}^{2 \pi} d \sigma e^{-i m \sigma}\left(\partial_{\tau} Y^{*} \partial_{\tau} Y+\partial_{\sigma} Y^{*} \partial_{\sigma} Y\right),
$$




$$
L_{m}^{\prime}=\frac{T}{2} \int_{0}^{2 \pi} d \sigma e^{-i m \sigma} T_{10}(0, \sigma)=\frac{T}{2} \int_{0}^{2 \pi} d \sigma e^{-i m \sigma} \partial_{\tau} Y^{*} \partial_{\sigma} Y .
$$

In quantum theory the substitution of modes gives:

$$
L_{n}=\frac{1}{2} \sum_{p=-\infty}^{\infty}\left(\alpha_{p}^{\mu^{*}} \alpha_{n+p}^{\mu}+b_{p}^{\mu} b_{n+p}^{\mu^{*}}\right)
$$

For elimination of the second term we will use again the $C$-symmetry requirements for each mode:

$$
\begin{aligned}
& L_{n p}=\frac{1}{2}\left(\alpha_{p}^{\mu^{*}} \alpha_{n+p}^{\mu}+b_{p}^{\mu} b_{n+p}^{\mu^{*}}\right)=\frac{1}{2}\left(\beta_{p}^{\mu^{*}} \beta_{n+p}^{\mu}+a_{p}^{\mu} a_{n+p}^{\mu^{*}}\right)=L_{n p}^{c}, \\
& \Lambda_{n p}=\frac{1}{2} \frac{1}{|p|}\left(\alpha_{p}^{\mu^{*}} \alpha_{n+p}^{\mu}-b_{p}^{\mu} b_{n+p}^{\mu^{*}}\right)=-\frac{1}{2} \frac{1}{|p|}\left(\beta_{p}^{\mu^{*}} \beta_{n+p}^{\mu}-a_{p}^{\mu} a_{n+p}^{\mu^{*}}\right)=-\Lambda_{n p}^{c},
\end{aligned}
$$

and obtain:

$$
b_{p}^{\mu} b_{n+p}^{\mu^{*}}=\beta_{p}^{\mu^{*}} \beta_{n+p}^{\mu} .
$$

These relations allow us to obtain the normal-ordered expression:

$$
L_{n}=\frac{1}{2} \sum_{p=-\infty}^{\infty}\left(\alpha_{p}^{\mu^{*}} \alpha_{n+p}^{\mu}+\beta_{p}^{\mu^{*}} \beta_{n+p}^{\mu}\right) .
$$

For the finding an algebra of operators further we use following relations:

$$
\begin{aligned}
{\left[\alpha_{m}^{\mu}, \alpha_{n}^{\nu^{*}}\right] } & =-|m| \eta^{\mu v} \delta_{m n}, \quad\left[\alpha_{m}^{\mu}, \alpha_{n}^{v}\right]=\left[\alpha_{m}^{\mu^{*}}, \alpha_{n}^{\nu^{*}}\right]=0, \\
{\left[\alpha_{m}^{\mu}, L_{n}\right] } & =\sum_{p=-\infty}^{\infty}\left[\alpha_{m}^{\mu}, \alpha_{p}^{v^{*}} \alpha_{n+p}^{v}\right]=\sum_{p=-\infty}^{\infty}\left[\alpha_{m}^{\mu}, \alpha_{p}^{v^{*}}\right] \alpha_{n+p}^{v}= \\
& =-|m| \sum_{p=-\infty}^{\infty} \eta^{\mu v} \delta_{m p} \alpha_{n+p}^{v}=-|m| \alpha_{m+n}^{\mu}, \\
{\left[\alpha_{m}^{i^{*}}, L_{n}\right] } & =\sum_{p=-\infty}^{\infty}\left[\alpha_{m}^{i^{*}}, \alpha_{p}^{j^{*}} \alpha_{n+p}^{j}\right]=\sum_{p=-\infty}^{\infty} \alpha_{p}^{\nu^{*}}\left[\alpha_{m}^{\mu^{*}}, \alpha_{n+p}^{v}\right]= \\
& =|m| \sum_{p=-\infty}^{\infty} \alpha_{p}^{\nu^{*}} \eta^{\mu v} \delta_{m, n+p}=|m| \alpha_{m-n}^{\mu^{*}} .
\end{aligned}
$$

Similar relations take place and for the $C$-conjugate operators also. Further we obtain: 


$$
\begin{aligned}
& {\left[L_{m}, L_{n}\right]=\sum_{p=-\infty}^{\infty}\left[L_{m}, \alpha_{p}^{\mu^{*}} \alpha_{n+p}^{\mu}\right]+\ldots=} \\
& =\sum_{p=-\infty}^{\infty}\left[L_{m}, \alpha_{p}^{\mu^{*}}\right] \alpha_{n+p}^{\mu}+\sum_{p=-\infty}^{\infty} \alpha_{p}^{\mu^{*}}\left[L_{m}, \alpha_{n+p}^{\mu}\right]+\ldots= \\
& =-\sum_{p=-\infty}^{\infty}|p| \alpha_{p-m}^{\mu^{*}} \alpha_{n+p}^{\mu}+\sum_{p=-\infty}^{\infty}|n+p| \alpha_{p}^{\mu^{*}} \alpha_{m+n+p}^{\mu}+\ldots= \\
& =-\sum_{p=-\infty}^{\infty}|p| \alpha_{p-m}^{\mu^{*}} \alpha_{n+p}^{\mu}+\sum_{p=-\infty}^{\infty}|p+n-m| \alpha_{p-m}^{\mu^{*}} \alpha_{n+p}^{\mu}+\ldots= \\
& =\sum_{p=-\infty}^{\infty}(|p+n-m|-|p|) \alpha_{p-m}^{\mu^{*}} \alpha_{n+p}^{\mu}+\ldots= \\
& =|n-m| \sum_{p=-\infty}^{\infty}\left(\alpha_{p}^{\mu^{*}} \alpha_{n+m+p}^{\mu}+\beta_{p}^{\mu^{*}} \beta_{n+m+p}^{\mu}\right)=|n-m| L_{n+m} .
\end{aligned}
$$

Thus, the required algebra of operators is given by the relations:

$$
\left[L_{m}, L_{n}\right]=|n-m| L_{n+m} .
$$

As we see, at the absence of the zero-point energy of string modes, in these commutators there are no anomalous terms.

\section{Conclusion}

We conclude that due to the chiral symmetry the relativistic boson string modes do not contain the zero-point energy. At the lack of the zero-point energy of modes and automatically normal ordering, the algebra of operators is normal (there is no central extension in the Virasoro algebra) and anomaly does not arise. For the same reason at quantization of a fermion string, where a chiral symmetry takes place also, the anomaly does not arise and there is no zero-point energy.

The lack of conformal anomaly does not allow to fix dimensionality $D$ of spacetime into which the string is embedded and the string theories can be consistently quantized at arbitrary dimensional spacetime $D>2$.

\section{References}

1. Zakir Z. Theor. Phys., Astrophys. and Cosmol., (2007) 2, 2, 9; 2, 3, 22; (2008) 3, 1, 1. 